\title{
1 The MAPS-based vertex detector for the STAR Experiment: \\ 2 lessons learned and performance
}

3 Giacomo Contin ${ }^{1}$ for the STAR collaboration

$4{ }^{1}$ Lawrence Berkeley National Laboratory, Berkeley, CA 94720, USA

5 E-mail:gcontin@lbl.gov

6 Abstract:

7 The PiXeL detector (PXL) of the STAR experiment at RHIC is the first application of the state-of-the-art thin 8 Monolithic Active Pixel Sensors (MAPS) technology in a collider environment. The PXL, together with the 9 Intermediate Silicon Tracker (IST) and the Silicon Strip Detector (SSD), form the Heavy Flavor Tracker (HFT), which has been designed to improve the vertex resolution and extend the STAR measurement capabilities in the heavy flavor domain, providing a clean probe for studying the Quark-Gluon Plasma.

The two PXL layers are placed at a radius of 2.8 and $8 \mathrm{~cm}$ from the beam line, respectively, and is based on ultrathin high resolution MAPS sensors. The sensor features $20.7 \mu \mathrm{m}$ pixel pitch, $185.6 \mu \mathrm{s}$ readout time and $170 \mathrm{~mW} / \mathrm{cm}^{2}$ power dissipation. The detector is air-cooled, allowing a global material budget of $0.4 \%$ radiation length on the innermost layer. A novel mechanical approach to detector insertion allows for fast installation and integration of the pixel sub detector. The HFT took data in Au+Au collisions at $200 \mathrm{GeV}$ during the 2014 RHIC run. Modified during the RHIC shutdown to improve its reliability, material budget, and tracking capabilities, the HFT took data in $\mathrm{p}+\mathrm{p}$ and $\mathrm{p}+\mathrm{Au}$ collisions at ${\sqrt{\mathrm{S}_{\mathrm{NN}}}}=200 \mathrm{GeV}$ in the 2015 RHIC run.

In this paper we present detector specifications, experience from the construction and operations, and lessons learned. We also show preliminary results from $2014 \mathrm{Au}+\mathrm{Au}$ data analyses, demonstrating the capabilities of charm reconstruction with the HFT.

\section{Contents:}

1. Introduction .

2. The MAPS-based PXL detector 


\section{1. Introduction}

37 The PiXeL detector (PXL) of the STAR Experiment at the relativistic Heavy lon Collider (RHIC),

38 Brookhaven National Laboratory (BNL), is the first vertex detector based on the state-of-the-art MAPS

39 technology to be operated in a collider environment. The PXL detector, together with the Intermediate

40 Silicon Tracker (IST) and the Silicon Strip Detector (SSD), is part of the Heavy Flavor Tracker (HFT), which

41 has been designed to extend the measurement capabilities of the STAR Experiment in the heavy flavor

42 domain [1][2]. Heavy quark measurements are a key component for the systematic characterization of

43 the dense medium created in heavy ion collisions, the so-called Quark-Gluon Plasma (QGP), which is one

44 of the main goals of the STAR Experiment heavy ion program.

45 The STAR experiment uses a Time Projection Chamber (TPC) inside a $0.5 \mathrm{~T}$ magnetic field as its main tracking detector. With its $1 \mathrm{~mm}$ pointing resolution at the vertex, the TPC is not able to distinguish the decay vertices of short-lived heavy flavor particles, like $D^{0}$ mesons $(c \tau \sim 120 \mu \mathrm{m})$, in the high multiplicity environment typically produced in $\mathrm{Au}+\mathrm{Au}$ collisions at $\mathrm{Vs}_{\mathrm{NN}}=200 \mathrm{GeV}$. The HFT, consisting of 4 cylindrical silicon detector layers, has been inserted inside the TPC inner field cage in 2014 to improve the track pointing resolution of the STAR detector (see Figure 1). The outermost layer of the HFT is placed at $22 \mathrm{~cm}$ from the beam line and is equipped with the SSD: it is based on double sided silicon strip sensors with $95 \mu \mathrm{m}$ inter-strip pitch and $35 \mathrm{mrad}$ relative $\mathrm{P}-\mathrm{N}$-side stereo angle; the SSD silicon and front-end chips were part of an existing detector [3] and have been equipped with new faster electronics, allowing for a data acquisition rate up to $1 \mathrm{kHz}$. The IST is placed at $14 \mathrm{~cm}$ radius and based on single-sided silicon pad sensors with $600 \mu \mathrm{m} \times 6 \mathrm{~mm}$ pitch. The two innermost PXL layers are placed at 8 and $2.8 \mathrm{~cm}$ radius. In order to achieve the required track pointing resolution, STAR tracks inward with graded resolution from the TPC (resolution $\sim 1 \mathrm{~mm}$ ) to the vertex, by using the SSD and the IST intermediate resolution $(\sim 250-300 \mu \mathrm{m})$ to guide the tracks to the two innermost layers of PXL ( 30 $\mu \mathrm{m})$.

59 The PXL detector design specifications, construction and expected performance are described in the 60 following section of the paper. Section 3 describes the operational issues experienced during the first 61 two years of data taking. The measured performance of the PXL detector and its contribution to the 62 physics measurement of STAR are reported in Section 4. 




Figure 1: Structure of the STAR HFT. The HFT consists of a three detector upgrade inserted in the TPC inner field cage.

\section{The MAPS-based PXL detector}

\subsection{The Ultimate-2 sensor} The PXL detector uses Ultimate-2, a sensor based on CMOS Monolithic Active Pixel Sensor (MAPS) technology, developed by IPHC in Strasbourg, France and optimized for the STAR experiment [4][5]. These sensors, thinned down to $50 \mu \mathrm{m}$ to reduce their contribution to the material budget, use pixels with a pitch of $20.7 \mu \mathrm{m}$ arranged in a 928 (rows) by 960 (columns) array (almost 1 Megapixel per sensor) on a $(20.22 \times 22.71) \mathrm{mm}^{2}$ chip with a high-resistivity epitaxial layer to increase the radiation hardness and improve the signal-to-noise performance. The total signal for a MIP has been measured to be 1000 e-, with a signal-to-noise ratio of about 30 for the pixel collecting most of the charge in the cluster. Each pixel includes readout and correlated double sampling (CDS) circuitry for signal extraction and noise subtraction. The reticle is divided into 4 sub-arrays to allow the process variation compensation with independent configurations of the reference voltages. The pixel array is read out by addressing one row at a time and processing all columns in parallel through programmable threshold discriminators located at the end of each column. The integration time of the whole sensor is $185.6 \mu$ s. The resulting digital data are then passed through a zero-suppression logic block located at the periphery of the pixel array on the same chip, which delivers encoded hit addresses for up to a maximum of 9 hit clusters per row; the data are then passed to on-chip memory intermediate buffering. The memory is arranged in two banks of 1500 words each allowing simultaneous read and write operations. The data are read out bit- serially from one of these memory banks over two Low-Voltage Differential Signaling (LVDS) outputs per sensor, each running at $160 \mathrm{MHz}$. Most of the sensor internal parameters can be programmed with a serial interface using the JTAG protocol. A low power dissipation of $170 \mathrm{~mW} / \mathrm{cm}^{2}$ allows these sensors 
87 material budget. The relatively short integration time $(185.6 \mu \mathrm{s})$ and the radiation tolerance (up to $880.9 \mathrm{kGy} /$ year and $2 \times 10^{11}$ to $10^{12} 1 \mathrm{MeV} \mathrm{n}_{\text {eq }} / \mathrm{cm}^{2}$ ) meet the specifications imposed by the STAR 89 experimental conditions.

\section{$90 \quad$ 2.2 The PXL construction}

91 The PXL detector has been designed as a highly parallel system [6]. It is subdivided into 2 detector-

92 halves attached on one side to a unique cantilevered support mechanics, allowing for fast insertion 93 and retraction of the detector by manually sliding the detector-halves along rails inside a support cylinder and locking them into a reproducible position using kinematic mounts. Each half consists of 5 sectors mounted in dovetail slots. A sector represents the basic unit in terms of powering and readout and consists of a trapezoidal thin $(250 \mu \mathrm{m})$ carbon fiber sector tube with four 10-sensor ladders mounted on each tube, one at the inner radius, and 3 at the outer radius, arranged in a turbo geometry design (see Figure 2).

After being pre-diced through deep-reactive-ion-etching (DRIE) technique, thinned by grinding and diced, the sensors, fully probe tested and characterized, are positioned with butted edges on flex cables using acrylic adhesive and precision vacuum chucks [7]. The sensors and the first electronics board responsible for power distribution and signal buffering are electrically connected via standard wire bonding to the flex cable and the wires are encapsulated for protection. The structure is stiffened by a carbon fiber backer glued at the bottom of the flex cable. At this point, precision vacuum chucks are used to attach the ladders to the sector tubes. The ladder and sector assembly fixtures rely on a series of pins and holes for the alignment of the different components. Fully assembled sectors are surveyed in a coordinate measurement machine (CMM) with an accuracy of about $5 \mu \mathrm{m}$ : the position of fiducial markers on the silicon sensors and the ladder surface profile are mapped and related to a set of 3 tooling balls mounted on the sectors. In this way a full map of the pixel positions on each sector is achieved. The completed sectors are then assembled into detector halves which are surveyed to form completely mapped stable units with the pixel positions known to an accuracy of approximately $10 \mu \mathrm{m}$. The ladder functionalities are tested after each assembly and position measurement step. Finally, the detector halves are attached to the insertion mechanics completing the detector assembly in novel approach to insertion mechanics allows replacing it with a spare detector copy within one day without interrupting beam operations [8]. 




Figure 2: PXL half-detector: 5 sectors mounted in dovetail slots on the carbon fiber support and connected to the insertion mechanics.

\subsection{The HFT track pointing resolution}

The excellent pointing resolution of the HFT is driven by the unprecedented characteristics of the PXL, that with a small inner layer radius, high granularity and low material budget limits the Multiple Coulomb Scattering in the detector. By design, the total track pointing resolution, separated into the geometrical and Multiple Coulomb Scattering components, has been evaluated to be $10 \oplus 24 \mathrm{GeV} / \mathrm{p} \times \mathrm{c}$ $\mu \mathrm{m}$ for a particle with momentum $p$ and is compatible with the its measured value from the data of the 2014 Au+Au run.

The geometrical component of the track pointing resolution depends on the radii of the two layers and to the intrinsic single layer hit position resolution. The position resolution component due to the pixel pitch of the sensor results in about $6 \mu \mathrm{m}$ and can be improved to $3.6 \mu \mathrm{m}$ by cluster centroid reconstruction methods. The effect of the cooling air flow on the detector position has been measured with a capacitive probe showing a stable displacement of $\sim 30 \mu \mathrm{m}$ with $\sim 5 \mu \mathrm{m}$ RMS vibrations at full flow. The displacement due to the thermal expansion at operating temperature has been also measured to be $\sim 5 \mu \mathrm{m}$. These contributions result in an overall single layer hit position resolution of $\sim 6.2 \mu \mathrm{m}$.

The Multiple Coulomb Scattering component of the track pointing resolution mainly depends on the material budget of the innermost layer. A major effort has been made to minimize the material budget of the PXL detector, achieving $0.39 \% X_{o}$ on the inner layer and $0.49 \% X_{o}$ on the outer layer, as evaluated from the characteristics of the materials and the assembly procedure (see Figure 3 ). Moreover, due to the low power consumption of the Ultimate- 2 sensor, a simple air cooling system is sufficient avoiding the additional material introduced by a liquid cooling system. The contribution of the silicon to the material budget has been reduced to $0.053 \% X_{o}$ thinning the silicon wafers to $50 \mu \mathrm{m}$ thickness with a controlled grinding process. Once thinned, the sensors assume their typical curved shape which makes handling and probe testing very challenging. A commercial probe testing system specifically optimized for such thinned sensors: a vacuum chuck has been designed to hold in a flat position up to 20 sensors 
simultaneously, to allow automated testing. The probe pins are mounted on a dedicated electronics card and designed to deal with the uneven pad ring surface, they have been optimized to allow for additional overdrive distance to improve contact reliability. The total sensor production yield, including thinning, dicing and probe testing, varied between $40 \%$ and $60 \%$, mainly depending on the quality of the contact achieved by the probe card in use. The flex conductive cable, based on a conductor-kaptonconductor layout, contributes to the material budget with $0.128 \% X_{o}$ in the version adopted for the innermost layer, featuring two 32 um-thick layers of aluminum, and with the $0.232 \% X_{o}$ for the copper version installed on the outer layer. A relevant fraction of it $\left(0.070 \% X_{0}\right)$ is represented by the encapsulant used to protect the wire bonds. The ladder design includes also an acrylic adhesive layer, preventing damages arising from different thermal expansion characteristics. The carbon composite used to fabricate the $125 \mu \mathrm{m}$ thick ladder stiffener and the $250 \mu \mathrm{m}$ thick sector support tube adds

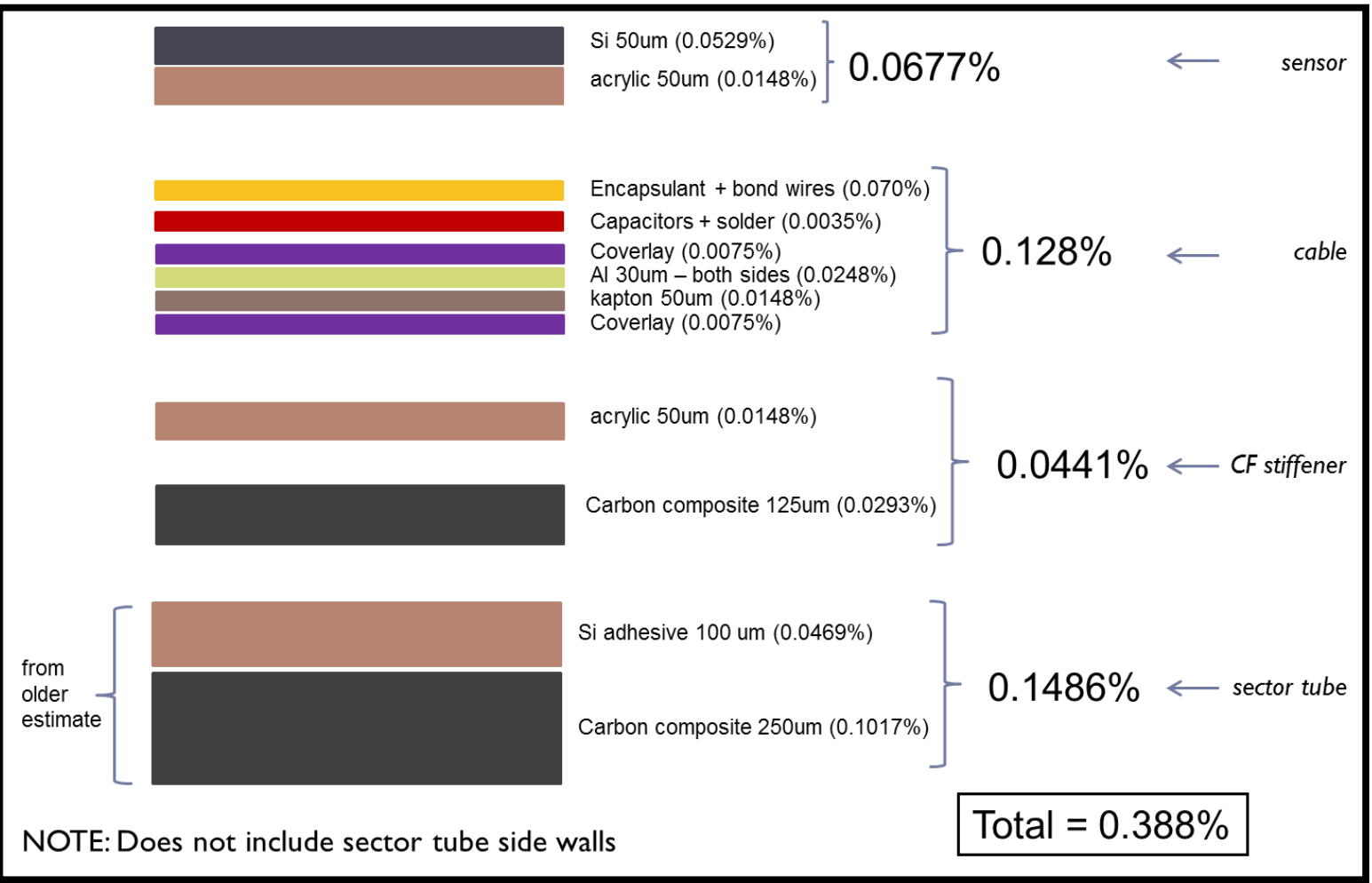

Figure 3: Contributions to the material budget for the PXL inner layer.

\section{Lesson learned: latch-up induced damage}

159 The PXL detector was installed in STAR and commissioned in January 2014. During the first weeks of Au+Au collisions in 2014 data taking, the detector started to show latch-up related sensor damage in many different forms: increased digital current consumption, damage to pixel columns, loss of full or partial pixel sub-arrays. Most of the failures occurred in the inner layer and only some of the outer layer were affected. A total of 16 of the 400 sensors in the PXL detector were damaged in the initial period, corresponding to a loss of $14 \%$ of the active surface on the inner layer and $1 \%$ on the outer layer. This limited damage did not avoid the successful completion of the 2014 physics run. 
The failure mechanism has been extensively studied through in a test campaign carried out in Fall 2014 at the 88" Cyclotron BASE Facility at the Lawrence Berkeley National Laboratory (LBNL) where existing PXL ladders and sensors have been exposed to heavy ion and proton beam irradiation. This test allowed the characterization of the damage mechanism and for the definition of a safe operation procedure to set up the power supply current threshold settings for the subsequent data taking periods.

The sensors tested included $50 \mathrm{~m}$ and $700 \mathrm{~m}$ full thickness devices with both high and low resistivity epitaxial layers. Current limited latch-up states have been observed with a typical increase of $300 \mathrm{~mA}$ in the operating digital current. Damage similar to the one observed in the STAR environment, with permanent increase of operating digital current and sensor data corruption, has been reproduced only on thinned high-resistivity sensors. An over-current protection threshold at $80 \mathrm{~mA}$ above the operating current ( $1 \mathrm{~A}$ ) was applied to the running detector and limited significantly the onset of latch-up induced damage to only 3 sensors on the inner layer in the entire 2015 Run. A further analysis of the damaged sensors, with an infrared camera, located the damage in specific structures of the digital section. The sensor substrate and sensitive epi-layer in the region of interest have been removed via plasma etching technique and examined through Scanning Electronic Microscope at the BNL Instrumentation Division laboratories, showing a modification of the metal layer, which appears to be melted and then affecting the sensor operational behavior.

\section{Status and performance of the HFT}

After being successfully commissioned, the HFT collected $\sim 1.2$ Billion minimum-bias Au+Au events during the 2014 Run and 1 Billion p+p plus 0.6 Billion p+Au minimum-bias events in the 2015 Run, at a typical trigger rate of about $0.8 \mathrm{kHz}$ with a dead time lower than $5 \%$.

The SSD and IST subsystems have been operated in stable conditions for most of the first two years of operations, accounting for $80 \%$ and $95 \%$ active channels respectively. The PXL detector was assembled and installed every year with an active channel fraction larger than 99\%. Due to the radiation environment, the PXL detector ended the 2015 physics data taking period with $5 \%$ inactive sensors on each layer. The sensor discriminator thresholds are calibrated in order to obtain an accidental noise hit rate of $\sim 1.5 \times 10^{-6}$. The measured characteristics of the sensors demonstrated that this configuration preserves a detection efficiency larger than 99\%, even after exposure to an ionizing radiation dose of $150 \mathrm{kRad}$ and non-ionizing radiation dose of $3 \times 10^{12} 1 \mathrm{MeV}$ neq $/ \mathrm{cm}^{2}$ [9]. The maximum hit multiplicity recorded by the PXL detector when taking data was of 400 hits per event per sensor on the inner layer and 70 on the outer layer. The over-current protection now implemented triggers on average $\sim 2$ reset cycles per minute for the full system, corresponding to 3\% dead time.

In the 2014 Run, the first PXL detector featured aluminum conductor only on two inner ladders, due to the technical difficulties that delayed the production of the double-sided aluminum flex cables. The remaining 38 ladders were assembled using copper conductor. All the sectors have been equipped with aluminum-conductor flex cables on the inner layer for the following data taking periods (2015 Run, 2016 Run). After the installation the PXL sectors have been aligned using cosmic ray data with resolution better than $25 \mu \mathrm{m}$. The analysis of $\mathrm{Au}+\mathrm{Au}$ data collected in 2014 Run demonstrated that the track pointing resolution of the HFT system meets the design requirements, achieving $\sim 46 \mu \mathrm{m}$ for $750 \mathrm{MeV} / \mathrm{c}$ 
Kaons for the 2 sectors equipped with aluminum cables on inner layer, and better than $30 \mu \mathrm{m}$ for particle momenta larger than $1 \mathrm{GeV} / \mathrm{c}$ (see Figure 4). This performance enabled the study of the Dmeson productions with high significance signal and the measurement of observables like their nuclear modification factor $R_{A A}$ [10] and collective flow $v_{2}$ [11]. A preliminary measurement of the $D^{0} \rightarrow K \pi$ production in $V \mathrm{~s}_{\mathrm{NN}}=200 \mathrm{GeV} \mathrm{Au}+\mathrm{Au}$ collisions from a partial event sample is shown in Figure 5.



Figure 4: Track pointing resolution along the beam coordinate as a function of the particle momentum, as measured for the two sectors equipped with aluminum conductor flex cables on the inner layer in $2014 \mathrm{Au}+\mathrm{Au}$ data.

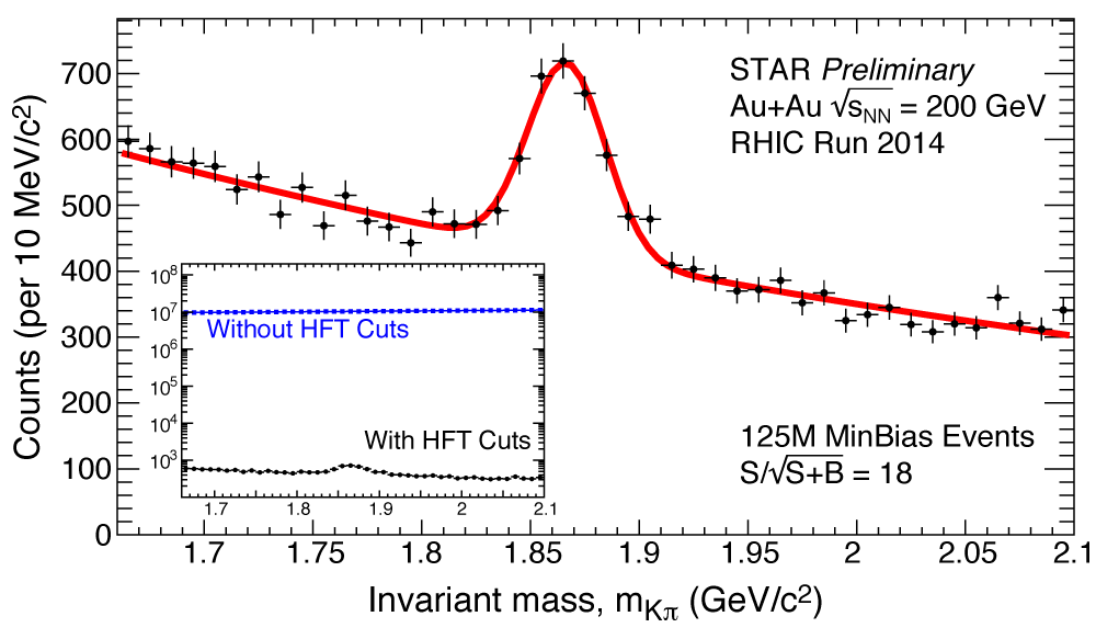

Figure 5: $D^{0} \rightarrow K \pi$ production in $v_{s_{N N}}=200 G e V$ Au+Au collisions, from a partial event sample of 125 Million minimum-bias events collected in 2014. The inset shows the suppression of the combinatorial background by $\sim 4$ orders of magnitude introduced by the HFT.

\section{Conclusions}

The PXL detector for the STAR Experiment demonstrates that the state-of-the-art MAPS technology is suitable for vertex detectors. As part of the Heavy Flavor Tracker (HFT), it successfully took data in $A u+A u, p+p$ and $p+A u$ collisions at $V s_{N N}=200 \mathrm{GeV}$ during the 2014 and 2015 RHIC runs. A latch-up induced damage on the PXL sensors has been observed, studied and limited through operational methods. The 2014 data analysis results show that the track pointing resolution performance of the HFT 
exceeds the design goals, thanks to the minimal material budget introduced by the innermost layers and single layer hit position resolution achieved. This performance enabled the study of the D-meson productions through topological decay reconstruction, extending the measurement capabilities of the STAR Experiment in the heavy flavor domain.

\section{Acknowledgments}

This work was supported by the Director, Office of Science, Office of Nuclear Science of the U.S. Department of Energy under Contract No. DE-AC02- 05CH11231. We gratefully acknowledge the PICSEL group of IPHC Strasbourg (M. Winter et al.) for the development of the PXL detector sensors.

\section{References}

[1] J. Adams et al., "Experimental and theoretical challenges in the search for the quark-gluon plasma: The STAR Collaboration's critical assessment of the evidence from RHIC collisions", Nucl. Phys. A 757 (2005), 102-183.

[2] C. Chasman et al., “A Heavy Flavor Tracker for STAR”, (2008), LBNL/PUB-5509-2008.

[3] L. Arnold et al., "The STAR silicon strip detector (SSD)", Nucl. Instrum. Meth. A499 (2003) 652-658 physics/0211083, doi:10.1016/S0168-9002(02)01963-0

[4] M. Winter, "Achievements and perspectives of CMOS pixel sensors for charged particle tracking", Nucl. Instrum. Meth. A 623 (2010), 192-194, doi:10.1016/j.nima.2010.02.192.

[5] C. Hu-Guo et al., "First reticule size MAPS with digital output and integrated zero suppression for the EUDET-JRA1 beam telescope", Nucl. Instrum. Meth. A 623 (2010), 480-482, doi:10.1016/j.nima.2010.03.043

[6] L. Greiner et al., "A MAPS based vertex detector for the STAR experiment at RHIC," Nucl. Instr. Meth. A 650 (2015) 68-72, doi:10.1016/j.nima.2010.12.006

[7] G. Contin et al., "The MAPS based PXL vertex detector for the STAR Experiment", JINST 10 (2015) C03026, doi:10.1088/1748-0221/10/03/C03026

[8] H. Wieman et al., "HFT PXL mechanics", Talk at the Forum on Tracking Detector Mechanics 30 June-2 July 2014 at DESY, Hamburg

[9] A. Dorokhov et al., "High-resistivity CMOS pixel sensors and their application to the STAR PIXEL detector", Nucl. Instrum. Meth. A 650 (2011), 174-177, doi: 10.1016/j.nima.2010.12.112

[10] G. Xie et al., "Nuclear Modification Factors of D Meson Production in Au+Au Collisions at $\vee_{\mathrm{s}_{\mathrm{NN}}}=200 \mathrm{GeV}$ ", Quark Matter 2015 in Kobe, Japan, Sep 2015

[11] M. Lomnitz et al., "Measurement of D Meson Azimuthal Anisotropy in Au+Au Collisions at $\sqrt{\mathrm{s}_{\mathrm{NN}}}=200 \mathrm{GeV}$ from STAR", Quark Matter 2015 in Kobe, Japan, Sep 2015 